\title{
Special Issue Editorial: Robotics: Mechanics and Control of Locomotion
}

\author{
Maurizio Porfiri ${ }^{1}$
}

Published online: 27 April 2017

(C) Springer Science+Business Media New York 2017

Robotics is transforming the way we progress as a society, creating technological opportunities that could have not been anticipated only a decade ago. For example, surgical robots are now routinely integrated in the medical practice, enabling doctors to perform complex procedures that would otherwise be hindered by traditional approaches. Similarly, unmanned vehicles are changing our capacity to access and monitor the environment, thereby fostering scientific discovery and engineering applications.

The main goal of this special issue is to present a coherent view into the potential of nonlinear science of informing and supporting robotics-related research. The collection of articles is largely focused around the mechanics and control of locomotion, an exemplary domain where nonlinear science naturally meets robotics. These articles are not intended to provide a comprehensive review of this exciting and active domain, but rather to demonstrate advancements in nonlinear science research in the areas of flying, swimming, and walking robots.

Spinello and Fattahi study the locomotion of a multi-legged robot inspired by millipedes and centipedes. The high redundancy and robustness of the locomotion of these organisms are known to be critical to their ability to successfully explore unstructured environments. The authors propose a distributed mechanical model, based on sheardeformable beam theory, to explain this complex form of multi-legged locomotion. Variational calculus is leveraged to establish a control-oriented mathematical formulation, which is illustrated through numerical simulations. Computational analysis

Communicated by Paul Newton.

$凶 \quad$ Maurizio Porfiri mporfiri@nyu.edu

1 Department of Mechanical and Aerospace Engineering, New York University

Tandon School of Engineering, Brooklyn, NY 11201, USA 
supports the feasibility of shape morphing and locomotion, offering important insight into geometrical and physical design.

Boyer et al. propose a unified framework for modeling and simulation of unconstrained locomotion of robots with soft or flexible appendages. This type of soft locomotion is also inspired from the animal world, where fish and insects routinely take advantage of compliance to save energy and perform complex maneuvers. Within the Newton-Euler modeling formalism of robotics, the paper puts forward a general theory for the study of mobile multibody systems, with body deformation and propulsion. The framework is demonstrated through the detailed study of passive swimming in a von Karman vortex street and hovering flight with flexible flapping wings.

Soft locomotion in a fluid is also the topic of investigation of Behbahani and Tan, who present a dynamic model for a robotic fish with flexible pectoral fins. Through the integration of a physically-based lumped mechanical model and blade element theory, the authors establish a predictive framework to study the effect of compliance on locomotion performance and mechanical efficiency. The model is validated through experiments on a robotic fish, featuring three dimensionally printed fins of varying compliance. At low fin-beat frequencies, flexible fins are found to offer advantages over rigid fins in speed and efficiency, but rigid fins ultimately outperform flexible fins at high frequencies. Notably, the authors demonstrate the existence of frequency values at which the speed performance or the mechanical efficiency can be optimized for a given fin flexibility.

Just as fluid mechanics plays a central role in the design of underwater propulsion, so it does in advancing our understanding of sensing. Colvert et al. establish a mathematical framework for shear flow detection, inspired by a class of aquatic organisms which respond to differences in the hydrodynamic signal instead of absolute values of the ambient flow. Toward the synthesis of decoding algorithms to infer flow properties from primitive measurements, the authors consider a shear flow where they place two sensors that output the difference in velocity between them. From the measurement of the velocity, they demonstrate the feasibility of solving the related nonlinear inverse problem, thereby uniquely determining the characteristics of the ambient shear.

Flapping flight is the topic of investigation of the paper by Tahmasian and Woolsey. Specifically, the authors address the problem of controlling the three dimensional motion of a biomimetic air vehicle through vibrational control and averaging. For high frequency and high amplitude forcing, the closed-loop dynamics of the vehicle leads to a mathematically tractable formulation which is leveraged for the design of a trajectory tracking control system in three dimensions. Through numerical simulations, the authors demonstrate robustness to uncertainty and success in flight in hover and in forward, climbing flight.

Perturbation methods are at the core of the article by Chakon and Or, which addresses the analysis of a twistcar toy vehicle. This highly underactuated model system has two axles of passive wheels and a single means of actuation, through the steering handlebar. For small-amplitude oscillatory inputs, the authors establish explicit expressions for the leading-order dynamics of the twistcar vehicle. These expressions afford the systematic analysis of the interplay between structural properties and actuation on the overall dynamics. Experimental results on a robotic prototype support the validity of some of the theoretical claims. 
Guo et al. further contribute to the area of control systems for flying robots. The authors address the practically challenging problem of designing and implementing a visual-servo control system for high-flying aerial vehicles that are tasked with assisting the navigation of low-flying vehicles underneath. An adaptive-repetitive control system is proposed to mitigate uncertainties in the camera's parameters and in the localization of the low-flying vehicle. Through numerical simulations and experimental observations, the authors demonstrate the feasibility of localization and trajectorytracking control of a low-flying quadcopter.

Control system design is also the objective of the paper by Nguyen and Leonessa, who put forward a rigorous mathematical framework for the characterization of adaptive output feedback control laws for a wide class of unknown linear systems. The approach relies on an adaptive predictor, which can anticipate the output of a system from the time history of its output and input. While the formulation is amenable to the implementation of a wide array of control algorithms, the authors specialize their approach to a forward Riccati differential equation, accounting for actuator amplitude and rate saturation constraints. Experimental results on pitch, elevation, and trajectorytracking of a helicopter prototype are presented to illustrate the theoretical approach.

Last, but not least, the work by Mummolo et al. presents a computational framework for the estimation of balanced and falling states of legged systems. The approach integrates joint-space and Cartesian-space dynamics toward a refined description of legged locomotion, which lends itself to a nonlinear optimization problem. An iterative algorithm is formulated to construct the stability boundary between balanced and falling states. The framework is demonstrated through the analysis of example systems with varying degree of complexity, namely, an inverted-pendulum based legged systems and a biped walking robot.

This collection demonstrates the importance of nonlinear science to the formulation of physically based predictive models for robotic systems, the systematic design of effective control algorithms for their autonomous operation, and the rigorous analysis of experimental and numerical data associated with their implementation. From perturbation methods to stability theory, from fluid dynamics to solid mechanics, and from nonlinear control to optimization, the papers highlighted in this special issue illustrate translational value of nonlinear science research in robotics.

Pushing the boundaries of robotics toward new, empowering, technological breakthroughs requires overcoming several technical challenges and, perhaps, questioning the foundations of the field. More importantly, it demands a truly interdisciplinary effort that brings together computational, experimental, and analytical research in science and engineering. We hope that this special issue will further ignite the interest of nonlinear science researchers in the field of robotics, and stimulate new exciting interdisciplinary collaborations. 